bioRxiv preprint doi: https://doi.org/10.1101/2021.10.22.465453; this version posted October 23, 2021. The copyright holder for this preprint (which was not certified by peer review) is the author/funder, who has granted bioRxiv a license to display the preprint in perpetuity. It is made available under aCC-BY-NC-ND 4.0 International license.

\title{
Dynamic Cell Imaging: application to the diatom Phaeodactylum tricornutum under
} environmental stresses

Houda Bey ${ }^{1 \#}$, Florent Charton ${ }^{2 \#}$, Helena Cruz de Carvalho ${ }^{2,3}$, Shun Liu ${ }^{2}$, Richard G. Dorrell ${ }^{2}$, Chris Bowler $^{2}$, Claude Boccara ${ }^{1 *}$, Martine Boccara ${ }_{2}^{1,2 *}$

1 Institut Langevin, ESPCI Paris, PSL Research University, CNRS UMR 7587, 1 rue Jussieu, 75005 Paris, France

2 Ecole Normale Supérieure, PSL Research University, Institut de Biologie de l'Ecole Normale Supérieure (IBENS), CNRS UMR 8197, INSERM U1024, 46 rue d'Ulm, F-75005 Paris, France

3 Université Paris Est-Créteil (UPEC), Faculté des Sciences et Technologie, 61, avenue du Général De Gaulle 94000 Créteil, France

\# These authors contributed equally to the work

- Corresponding authors, emails:

- mboccara@bio.ens.psl.eu

- claude.boccara@espci.fr 


\begin{abstract}
The dynamic movement of cell organelles is an important and poorly understood component of cellular organisation and metabolism. In this work we present a non-invasive nondestructive method (Dynamic Cell Imaging, DCI) based on light scattering and interferometry to monitor dynamic events within photosynthetic cells using the diatom Phaeodactylum tricornutum as a model system. For this monitoring we acquire few seconds movies of the signals that are related to the motion of dynamic structures within the cell (denoted scatterers), followed by a statistical analysis of each pixel time series. Illuminating P.tricornutum with LEDs of different wavelengths associated to short pulsed or continuouswave modes of illumination revealed that dynamic movements depend on chloroplast activity, in agreement with the reduction in the number of pixels with dynamic behaviour after addition of photosystemII inhibitors. We studied $P$. tricornutum under two environmentally relevant stresses, iron and phosphate deficiency. The major dynamic sites were located within lipid droplets and chloroplast envelope membranes. By comparing standard deviation and cumulative sum analysis of the time series, we showed that within the droplets two types of scatterer movement could be observed: random motions (Brownian type) but also anomalous movements corresponding to a drift which may relate to molecular fluxes within a cell. The method appears valuable for studying the effects of various environments on a large variety of microalgae in the laboratory as well as in natural aquatic environments.
\end{abstract}

\title{
KEYWORDS
}

Chloroplast/ Endoplasmic reticulum/ Light scattering/ Lipid droplets/ Iron limitation/Organelle movement/ Phosphate limitation.

\section{HIGHLIGHTs}

Light scattering an alternative to fluorescence to rapidly evidence dynamic processes. Lipid droplets the major metabolic active sites under stress A non-destructive visualisation method for laboratory microalgae and aquatic samples..

\section{SIGNIFICANCE STATEMENT}

Light scattering could be an alternative to fluorescence techniques to study dynamic processes within photosynthetic cells. We used a method combining light scattering and interferometry to analyse movements of intracellular scatterers in the marine diatom Phaedactylum tricornutum under two environmentally relevant stresses, iron and phosphate deficiency. Lipid droplets were the major active sites under stress. The method which is rapid and non destructive can be broadly expanded to study other microalgae and their stress responses, in the laboratory and in aquatic environments. 


\section{INTRODUCTION}

Diatoms are a diverse group of photosynthetic microorganisms, which account for up to $40 \%$ of ocean primary production (Bowler et al., 2010). They are distributed worldwide, from tropical and subtropical regions to polar ecosystems in oceans and fresh waters, and thus have an exceptional ability to adapt to highly dynamic aquatic environments (Falciatore et al., 2020; Pierella et al., 2020). These include stressful environmental fluctuations and chronic scarcities of key nutrients including nitrogen, phosphorus or iron (Abida et al., 2015 ; Alipanah et al., 2015; Cruz de Carvalho et al., 2016; Kazamia et al., 2018; Gao et al., 2021), extreme temperatures ranging from continuous near-freezing temperatures in ocean polar regions to hot currents in equatorial oceans (Yao et al. 2015; Liang et al 2019) or high light illumination levels (Domingues et al., 2012; Alboresi et al., 2016).

Diatoms have a complex evolutionary origin, which involved a secondary symbiotic event where a heterotrophic eukaryote engulfed a microalga of the red lineage (CavalierSmith, 1999). This has led to the presence of four membranes around the plastids of diatoms, with the outermost chloroplast membrane joined to the endoplasmic reticulum (ER) and several internal chloroplast compartments including the periplastid compartment (located between the second and third innermost membranes). To identify the metabolically active organelles in diatom cells under stress, methods involving organelle isolation, biochemical methods, genomic, and cell products characterisation have been very valuable (Abida et al., 2015; Lupette et al., 2019). Fluorescence imaging techniques with high spatial and temporal resolution have been previously used to further investigate dynamic processes in stressed diatoms (Lupette et al., 2019; Jaussaud et al., 2020). Indeed, cytological methods involving the use of specific stains or of fluorescent proteins are widespread methods used in microscopy. Green Fluorescent Protein (GFP) tagged proteins are used to visualize and track in real time proteins of interest as well as cell organelles (Apt et al., 2002; Kazamia et al., 2018). Among the many GFP applications, Fluorescence Recovery After Photobleaching (FRAP) has been used to characterize the transport of integral membrane proteins from the endoplasmic reticulum to lipid droplets in yeasts (Jacquier et al., 2011).

Studies of biological materials by Dynamic Cell Imaging (DCI) also called Dynamic Full field Optical Transmission Tomography (D-FFOTT) are based on the detection of light back-scattered generated by subcellular organelles (denoted scatterers) movements and do not need elaborate molecular manipulations, as is the case for GFP. This method is noninvasive and non-destructive (Apelian et al., 2016, Thouvenin et al., 2017, Scholler et al., 2019). A more recent variation of DCI, working in transmission and forward scattering,that 
we use here is described in Thouvenin et al., 2021. DCI was shown to be associated with metabolic activity as it disappeared when cells were fixed or treated with the metabolic inhibitor deoxy-glucose (Apelian et al., 2016). DCI appeared thus as a promising technique to study cell substructure dynamic in eukaryotic microorganisms unable to be genetically modified. For that reason we first studied the model unicellular photosynthetic microorganism Phaeodactylum tricornutum responding to nutrient stress conditions.

We studied two major nutrient stresses: iron and phosphate depletion. Iron $(\mathrm{Fe})$ is an essential micronutrient for of all living cells since it is crucial for photosynthesis and respiration, and for phytoplankton it is a cofactor of proteins involved in a number of metabolic reactions. Iron deficiency leads to a decrease in photosynthetic efficiency (Kolber et al., 1994) by inducing partial blocking of the electron transfer between photosystem II (PSII) and photosystem I (PSI) (Roncel et al., 2016). Iron deficiency is an important growthlimiting or co-limiting nutrient in many regions of the world's ocean in which diatoms are important primary producers (Ustick et al., 2021).

Phosphate is a fundamental element of all living cells being part of membranes, nucleic acids and other biomolecules. Phosphate limitation and co-limitation is frequently observed in low northern latitudes (Ustick et al., 2021). The deficiency of its inorganic form (Pi) is also a recurrent form of stress that highly limits ocean primary production. Indeed, inorganic phosphate $(\mathrm{Pi})$ deficiency is known to induce a shift in diatom metabolism leading to arrested cell growth and production of lipid vesicles (Cruz de Carvalho et al., 2016). It has recently been shown that these lipid vesicles (also known as lipid bodies or lipid droplets, accumulate in the vicinity of the chloroplasts (Alipanah et al., 2018; Lupette et al., 2019). Indeed limitation of nutrients such as nitrate and phosphate leads to remodelling of cellular membranes, with degradation of membrane phospholipids and fatty acids which are redirected to lipid bodies (Goss et al., 2020).

Various possible kinds of organelles movements can be observed in cells from a pure Brownian type to drifts with a defined velocity (Saunter et al., 2009). Here we characterized the slight amplitude movements of scatterers within P. tricornutum by analysing the time series associated to each pixel of the field of view in order to get a map of the transmission, the standard deviation, the cumulative sum and the average frequency. Through this computational analysis, we were able to highlight random and non-random driven movements suggesting that this method can spot in organello molecular fluxes.

\section{Material and Methods}




\section{Diatom culture conditions}

Phaeodactylum tricornutum CCMP632 was cultured in artificial sea water (40 g/L, Sigma) supplemented with f/2 nutrients and vitamins (F/2 Media Kit Bigelow NCMA) under continuous shaking $(100 \mathrm{rpm})$ at $20{ }^{\circ} \mathrm{C}$ under cool white fluorescent lights $\left(30 \mu \mathrm{E} \cdot \mathrm{m}^{-2} \mathrm{~s}^{-1}\right)$ with a $12 \mathrm{~h}$ photoperiod. For phosphate depletion studies equal aliquots of 4 day-old cultures from the same batch culture were inoculated in parallel in fresh $\mathrm{f} / 2$ media (control conditions) and in $250 \mathrm{~mL}$ fresh $\mathrm{f} / 2$ media without phosphate supplement (Pi depleted) and cultured in the same conditions as described above for 8 days.

For iron depletion, cultures were grown in enhanced seawater medium with or without iron-EDTA (supplemented concentration $86.9 \mu \mathrm{mol} / 1$ ), and analysed after two subcultures (with starting inoculum of 100,000 cells $/ \mathrm{ml}$ for 8 days. All cell lines were grown in ventilator-capped plastic culture flasks (Sigma) to minimise residual iron or phosphorus contamination from the culture flask into the growth media. Cells were collected by centrifugation and mixed with melted $1 \%$ agar in culture medium and immediately observed after jellification.

For photosystem II inhibition, we used 3-(3,4-dichlorophenyl)- 1,1-dimethylurea (DCMU; $40 \mu \mathrm{M}$ ) and hydroxylamine (HA; 2mM) solutions in water. Cells were incubated for 10 minutes at room temperature with inhibitors, embedded in agar and immediately observed.

\section{DCI: Data acquisition and treatment}

To follow the movement of internal structures within diatoms we used at the pixel level, the time variation of the optical tomographic images, the principle of which is described in detail in Apelian et al., (2016), Thouvenin et al., (2017) and Scholler et al., (2019) but using a new experimental approach, called Dynamic Full Field Optical Transmission Tomography (DFFOTT) that is described in detail in Thouvenin et al., 2021. In brief, the sample is illuminated in transmission by the incoherent light emitted by a LED; this beam is partially scattered by the sample structure and partially transmitted. Both beams propagate along the same path and interfere with a phase shift (known as the Gouy phase shift) that depends on the relative position of the scatterer and of the focus of the objective. The subcellular organelle movements induce a time variation of the signal detected on each pixel of the camera. We use a Photonfocus A1024B camera working at $140 \mathrm{~Hz}$ with a CMOS-imagesensor with a $0.2 \mathrm{Me}^{-}$full well capacity that insures shot noise limited measurements with a good signal-to-noise ration. 
To represent the evolution of signal over time we displayed a number of characteristic images extracted from a movie of typically 100 to 200 images: 1) The transmission image is analysed in order to check the stability of the cells and the lack of bleaching. 2) The average of standard deviation by group of one tenth of the movie in order to buffer any transient event. Steps 1 and 2 are run with Fiji (Schindelin et al., 2012). 3) The average cumulative sum by group of one tenth of the movie (Scholler, 2019) is compared to standard deviation of each pixel (voxel). Unlike a pure Brownian motion, a biased Brownian motion is hyperdiffusive. A way to distinguish the two types of motion is to compare the results of standard deviation to the cumulative sum. Indeed summing Brownian steps will give a trajectory that stays close to zero whereas if there is a small bias added to the Brownian random steps (figure S1), it will be summed for every sample and could be revealed by the cumulative sum; a more detailed description of this approach can be found in Scholler (2019). We used the cumsum function of Matlab which, when a small bias is present, is more adapted to reveal anomalous Brownian motion and, in addition increase the signal-to-noise ratio of the DCI. The normalization was made using the shot noise normal distribution in the background. All experiments have been repeated 3 times.

\section{Fluorescence microscopy}

We used several GFP (green fluorescent protein) constructions to characterize the dynamic location of several Phaeodactylum tricornutum organelles. C-terminal GFP constructs were assembled by Gibson assembly into pPhat vectors, and introduced into wild-type $P$. tricornutumm by biolistic transformation, followed by selection on zeocin-supplemented ESAW-agar plates, as previously described (Falciatore et al. 1999). We used, GFP with no targeting signal, here the signal accumulates in the cytosol. GFP fused to BiP protein (Binding immunoglobulin protein) signal peptide here GFP is localized in the ER (Apt et al 2002). GFP fused to the N-terminal extension comprising a signal peptide and a transit peptide of Hsp70 of Phaeodactylum tricornutum (Gould et al 2006) is expressed in the PPC (periplastid compartment). Full construct vector sequences are provided in Table S1. For all GFP experiments transgenic diatoms were analysed by standard inverted epifluorescence microscope (Nikon Ti-E) equipped with an oil immersion objective (90X, 1.4 NA) and an EMCCD camera (Andor Ixon Ultra X-10381). GFP was excited at $488 \mathrm{~nm}$ and emitted fluorescence detection was from 500 to $550 \mathrm{~nm}$. The sensitivity of the camera used for bright field recording (10 times lower full well capacity than the Photon Focus CMOS camera) did 
not allow merging of the two types of signal (fluorescence and interferometry) using the same field of view.

We also used multimodal imaging combining interferometry and fluorescence in a single optical setup (described in Thouvenin et al. 2017) to compare the fluorescent labelling with the distribution of DCI signals within lipid bodies. Diatoms were incubated with BODYPY 505/515 (dissolved in DMSO; $0.1 \mu \mathrm{g} \mathrm{mL}^{-1}$ final concentration) for 30minutes and observed by DCI and fluorescence. In this system we used a LED source centered at $470 \mathrm{~nm}$ (M470L4, Thorlabs, Newton, NJ, USA) for the excitation and filtered with a bandpass filter centered on $475 \mathrm{~nm}$ with a bandwidth of $50 \mathrm{~nm}$ (Semrock FF02- 475 / 50-25). The interferometry signal was recorded on a custom camera (Quartz 2A750, Adimec). The emitted fluorescence was filtered with another bandpass filter centered on $540 \mathrm{~nm}$ (Semrock FF01-540 / 50-25) then imaged using a camera sCMOS (Hamamatsu Photonics). Excitation and fluorescence wavelengths were separated by a dichroic mirror at $506 \mathrm{~nm}$ (Semrock FF506-Di03-25).

Experiment has been repeated two times with similar results.

\section{RESULTS}

\section{DCI reveals the dynamics of diatoms organelles}

Diatoms grown in supplemented artificial seawater were embedded into agar in the same medium and immediately observed with the DCI set up (or D-FFOTT Thouvenin et al., 2021). The preparation was illuminated for a few seconds (pulsed light, 1ms, LED blue $455 \mathrm{~nm}$ ) at a few tens of $\mathrm{Hz}$ and a film recorded synchronously at the same frequency. We used pulsed illumination to freeze movements within cells and to avoid light saturation of photosynthesis. When observing the film of the transmission, no overall movement was detected and the diatoms looked still (movie S1). When we computed the standard deviation of each voxel for all the frames of the film we observed two sites of movement within the cells: vesicles or droplets and the chloroplast membrane. The following figures display the cells metabolic activity (Apelian et al., 2016) the colour map code (ImageJ, 16 colours) is such that red corresponds to the highest activity and dark blue to the lowest activity that is linked to the speed of the scatterers movements. In the vicinity of the chloroplast, vesicles or lipid droplets of different sizes, appeared green, yellow and red with the most dynamic regions located at edges of the droplet (figure 1A, red arrow). The other highly dynamic location corresponded to the chloroplast membranes and often looked punctuated (figure 1A, white arrows). Interestingly, these results were observed independently of the three 
morphotypes that were present in the cultures (fusiform, triradiate, or oval) (De Martino et al., 2007). P. tricornutum cells were also illuminated with a green LED (505nm) or a deep red LED $(735 \mathrm{~nm}$, a spectral region where no P. tricornutum pigments absorb and no photosystems are excited). We observed with the green LED dynamic region surrounding the chloroplast and strong signal at the edges of the vesicles very similar to what we detected with the 455nm LED (figures 2, S2A). Histogram profiles of the standard deviation of signal intensity of each pixel were very similar between blue and 505nm LEDs with a shoulder corresponding to the strongest signals (figure S2B)). When we used the 735nm LED the signal was significantly reduced about 3 times (figures 2, S2A, S2B). We used two inhibitors at concentrations inhibiting photosystem II: 3-(3,4-dichlorophenyl)- 1,1-dimethylurea (DCMU; $40 \mu \mathrm{M}$ ) or hydroxylamine (HA; $2 \mathrm{mM}$ ) and measured the number of pixels with dynamic behaviour. We observed a significant reduction of about 3 times in presence of the inhibitors suggesting that the dynamic structures we described depend on chloroplast metabolic activity (figure 2). To further investigate this observation, we compared image acquisition between continuously or pulsed LED lighting (figure 1). No obvious dynamic structures within the cell were highlighted when illumination was continuous unlike with pulsed light although no difference in transmission was observed meaning that no photobleaching was involved (figures 1A and 1B). This suggested that the high continuous light photon budget prevents chloroplast function unlike the pulsed mode level of irradiation (average power 30 times lower, figures 1B) and is in good agreement with the photon budget given by Prins et al., (2020). Our observations suggest that during the continuous exposure to light, chloroplasts are likely saturated with photons, which results in the low metabolic activity recorded (Bailleul et al., 2011). We reasoned that the elimination of a harmful excess energy could be dissipated through heat, however using a micro-thermocouple immersed in the sample cuvette (volume $5 \mu \mathrm{l}$ ) we did not detect an overall measurable increase in temperature $\left(<0.1^{\circ} \mathrm{C}\right)$ under the same illumination level.

\section{Fluorescence studies}

To better characterize the cell compartments of the dynamic structures we described, Cterminal GFP fusions of proteins targeted to various compartments within P. tricornutum cells and observed with a fluorescent microscope (same samples analysed with DCI). GFP with no targeting signal led to its localization in the cytosol (figure S3, CYT). GFP fused to a bipartite targeting signal (signal sequence and transit peptide) of HSP70 enabling this protein to cross the two chloroplastic membranes, did not label the vesicles suggesting that they were 
not associated to periplastidal compartment (PPC) (figure S3, PPC). The signal sequence of BIP protein fused with GFP resulting in fluorescence in the endoplasmic reticulum (ER) labelled the dynamic vesicles (figure S3, ER). These observations suggested that vesicles possibly lipid droplets (see below) originate from the endoplasmic reticulum as already suggested by Jaussaud et al. (2020).

We took advantage of a setup which combined interferometry and fluorescence (Thouvenin et al. 2017) to compare the location of dynamic signals with those identifying lipid bodies. For this purpose we labelled lipid bodies with BODIPY505/515 and as shown in figure 3 we observed co localization of dynamic signals and lipid labelling. In addition we showed in other cells that dynamic signals lighted up more structures than the specific signal observed by fluorescence (figure S4)

\section{Dynamic of organelles in diatoms grown in iron-limited conditions}

Kazamia et al. (2018) demonstrated that iron uptake in Phaeodactylum when grown in iron-limited conditions, involved an endocytosis step and a delivery step in the vicinity of the chloroplast. This study suggested dynamic events in Phaeodactylum cells when iron is limited. We thus visualized the effect of iron limitation on $P$. tricornutum intracellular dynamics after one week and two weeks of culture. After one week of culture in irondepleted medium, a slight decrease in growth was observed suggesting a growth slow-down and after two weeks a ten-fold growth reduction was observed (Table 1). Samples were analysed using DCI after one (T1) or two (T2) transfers of culture with iron or iron-depleted media. In the presence of iron in the culture medium, we did not observe much difference between the two subcultures with dynamic pixels localized mostly in lipid droplets. We computed the number of pixels after removing the background noise of the same stack and found no differences between the two measurements (Table 1, figure S4). After one week of culture in iron-limited medium we observed a slight reduction (about $10 \%$ ) in the number of pixels with dynamic behaviour. After two weeks of culture in iron-limited medium the number of pixels with dynamic behaviour were $75 \%$ reduced (Table 1, figure S4). The pixels with dynamic behaviour were localized in the lipid droplets however on average there was no significant difference in the droplets diameters between the two conditions, with and without iron $(1.1 \pm 0.5 \mu \mathrm{m}$ and $0.9 \pm 0.2 \mu \mathrm{m}$ respectively). All these results suggest that in the iron-depleted medium the accumulation of lipids in droplets is arrested after prolonged starvation possibly due to the shutdown of photosynthetic carbon assimilation and/or lipid synthesis. 


\section{Dynamics of lipid bodies in diatoms subjected to phosphate deprivation}

It has been previously reported that after 8 days of culture in full nutrient-supplied media small lipid droplets start forming in P. tricornutum cells, suggesting the occurrence of a developing nutrient stress (Cruz de Carvalho et al., 2016). After 8 days in full culture media we observed on average, two lipid droplets per P. tricornutum cell and their diameters were computed (Table 2, figures $\mathbf{4 A}, \mathbf{S 5}$ ). The analysis of the standard deviation of signal intensity of each pixel showed that 30 to $50 \%$ of the droplets exhibited a dynamic behaviour (Table 2, figures 4A, S5). In the case of $P$. tricornutum grown for 8 days in phosphatedepleted medium there was a net reduction in cell growth (2 to 3 times) (Cruz de Carvalho et al., 2016) and a net increase in the number of droplets (5 to 10), which were significantly larger than when grown in Pi-replete medium (Table 2). Furthermore, the majority of the lipid droplets presented a dynamic behaviour (Table 2, figures 4B, S5). Generally the droplets were in close association with the chloroplast but sometimes they were found free in the cytoplasm and were able to fuse giving rise to very large structures (red arrow, figure S5). Histogram profiles showed a net increase (about 5 fold) in the number of pixels above the background noise between the conditions with and without phosphate (Table 3, figures 4, S5).

To get more insights into the dynamics of lipid droplets we analysed the time series of each pixel in order to get a map of the transmission, the standard deviation, the cumulative sum (Matalb: cumsum function) and the average frequency. Despite a noticeable difference in signal level (standard deviation and cumulative sum, see below) between the cells grown with $\mathrm{Pi}$ and without $\mathrm{Pi}$, we did not observe any significant differences with regard to the central frequencies, which were around $15+/-5 \mathrm{~Hz}$. We observed a 3-5 times increase in the signal amplitude (standard deviation and cumulative sum respectively) in P. tricornutum grown without phosphate (Table 3). The analysis of the signal value also pointed out also some differences of the signal distribution between standard deviation and cumulative sum computations. The computation of value variation of a pixel relied on the cumulative sum of the signals highlighted a non-Brownian displacement within the lipid droplets (Table 3). Following the method developed in detail in Scholler (2019) we found that the normalized cumulative sum is about two times that of the normalized standard deviation in culture with phosphate while it is slightly superior (1.2 times) in culture without phosphate (Table 3). This result suggests that the random movement of scatterers within the droplets is likely to be associated to a drift that makes it hyper-diffusive, rather than being a pure Brownian motion 
and could correspond to the flux of lipids and proteins filling lipid bodies (Olzmann and Carvalho 2019; Nettebrock and Bohnert 2020).

\section{DISCUSSION}

Here, we present a non-invasive and non-destructive method, which allows the detection and characterisation of internal movements within photosynthetic cells. In particular, through image analysis we show evidence that these dynamic processes are correlated with the functioning of the chloroplasts. This detection was possible by relying on the scattering properties of the cell organelles and of the high frame rate of our camera recordings. We were able to identify and quantify movements within lipid droplets, specific organelles, which accumulate in nutrient-stressed diatoms. In cells deprived of phosphate in addition to the random movement of little scatterers, which may correspond to the movement of membrane proteins from ER (Jacquier et al., 2011) or storage of misfolded proteins in lipid droplets as suggested by Lupette et al. (2019), we described a drift movement of scatterers, which might correspond to molecular fluxes of proteins and/or lipids. The distribution of dynamic pixels often observed in an eggcup shape (figure S6) could be in agreement with ER surrounding lipid droplets (Jacquier et al., 2011) and more precisely in the specific case of diatoms the most outer membrane of the chloroplast (Flori et al., 2016, Leyland et al., 2020, Jaussaud et al., 2020).

The DCI method can be extended to any microalgae considering that transformation is not available for many of them. We undertook a similar study with Fragilariopsis cylindrus grown under light or in dark and we observed many dynamic structures within this diatom (not shown). The method is very sensitive and allows the study of slight changes due to environmental fluctuations. In addition, most of image analyses have been run with Fiji (ImageJ), a public domain application (Schindelin et al., 2012).

Cytoplasmic streaming generated by myosin in the alga Chara or in plants has long been described (for review, Tominaga \& ilto, 2013). Indeed the speed of cytoplasmic streaming generated by Chara myosin observed by optical trap nanometry is in the range of $50 \mu \mathrm{m} / \mathrm{s}$ (Tominaga \& iIto, 2013). With DCI we observed life imaging of low speed movements (about $0.1 \mu \mathrm{m} / \mathrm{s}$ ) of undetectable scatterers within P. tricornutum organelles and corresponding to their metabolic activity. This method could be extended to other dynamic structures such as liquid droplets present in other organelles or in nuclei. 


\section{ACKNOWLEGEMENTS}

We wish to thank our colleagues: Ignacio Izzedine (Institut Langevin) for the use of the fluorescence microscope, Olivier Thouvenin (Institut Langevin) for his help in the dynamic signal analysis, and Benjamin Bailleuil for providing photosystem II inhibitors. HB and CB acknowledge financial support from the DIM ELICIT (INFLUERE IN VISCERA). FC acknowledges Q-Life for the PhD fellowship (Q-life ANR-17-CONV-0005), HCC acknowledges funding from the Agence National pour la Recherche (ANR DiaLincs 19CE43-0011-01), RGD acknowledges a CNRS Momentum Fellowship. CB acknowledges funding from the ERC (Diatomic project) and the ANR (Browncut).

\section{FUNDING}

\section{Funder}

DIM ELICIT

Q-Life

Agence Nationale

de la Recherche

$\begin{array}{ll}\text { CNRS } & \text { CNRS Momentum Fellowship } \\ \text { ERC } & \text { Diatomic project } \\ \text { ANR } & \text { Browncut }\end{array}$

\author{
Grant reference number \\ INFLUERE IN VISCERA \\ Q-life ANR-17-CONV-0005 \\ ANR DiaLincs 19-CE43-0011-01 \\ CNRS Momentum Fellowship \\ Browncut
}

\author{
Author \\ Houda Bey, Claude Boccara \\ Florent Charton \\ Helena Cruz de Carvalho \\ Richard Dorrell \\ Chris Bowler \\ Chris Bowler
}

\section{SUPPORTING INFORMATION \\ SUPPLEMENTARY TABLE \\ Table S1: list of primers for GFP constructs}

\section{SUPPLEMENTARY MOVIE}

Movie S1: Transmission film of diatoms illuminated at $455 \mathrm{~nm}$

\section{SUPPLEMENTARY FIGURES}

Figure S1: Simulation of a pure Brownian motion (blue) and of a biased movement (red) where a drift is added to the Brownian motion.

The $y$-axis of the graph shows. the dynamic movement of a particle) measured over time ( $\mathrm{x}$ axis). For this specific example the standard deviation was found to be 1.16 (Brownian) and 1.37 (Brownian biased) whereas the cumsum (cumulative sum) was found to be 12.30 (Brownian) and 180.64 (Brownian biased). ), i.e. demonstrating a the very large enhancement of the signal when a drift is present.

\section{Figure S2: Effect of wavelength illumination on intracellular movements in Phaeodactylum tricornutum}

Cells were successively illuminated with pulsed light from $\mathrm{LED}_{735}$ (red), $\mathrm{LED}_{505}$ (green) and $\mathrm{LED}_{455}$ (blue) and a film of few seconds recorded. The same field of view in the successive acquisition was selected; upper image: transmission; lower image standard deviation (scale on bottom left) (figure S2A). Histograms of the standard deviation images using image $\mathbf{J}$ (Fiji) were recorded and the number of pixels above noise level were computed (indicated by a red bar on the histogram) in figure S2B. Histograms of the same image for each 
wavelength, but without cells, were recorded to determine noise levels. Scale bar value was deduced from the format of the image $(60 \times 60 \mu \mathrm{m})$

\section{Figure S3: Co-localisation of dynamic lipid droplets from Phaeodactylum tricornutum and labelled endoplasmic reticulum}

Left panel: light microscopy, central panel: GFP fluorescence (grey scale camera, green coloration), right panel merge of the two upper images. Cytosolic (CYT) expressed GFP accumulates in the entire cell. The signal peptide of BIP protein fused to GFP leads to endoplasmic localization of GFP (ER). The dual topogenic signal of Hsp70 fused to GFP targets GFP to periplastid compartment (PPC). Scale bar value was deduced from the format of the image $(60 \times 60 \mu \mathrm{m})$.

\section{Figure S4: Dynamic signal in Phaeodactylum is not limited to lipid bodies}

Upper panel: transmission microscopy, central panel: Standard deviation (grey scale camera, 16 colors), lower panel merge of the two previous images. Scale bar value was deduced from the format of the image $(90 \times 90 \mu \mathrm{m})$.

\section{Figure S5: Decrease in dynamic signal in Phaeodactylum tricornutum grown in iron depleted medium}

Representative cells grown in medium with iron $(+\mathrm{Fe})$ or depleted of iron $(-\mathrm{Fe})$. From left to right panel is shown the transmission, the standard deviation of each pixel and the merged image (artificial blue colour). T1 and T2 correspond to 8 days and 16 days growth in medium with iron or depleted of iron. Histograms of the standard deviation images using image $\mathrm{J}$ (Fiji) were recorded and the number of pixels above noise level were computed (indicated by a red bar on the histogram) in Table 1. Histograms of the same image size without cells, were recorded to determine noise levels Scale bar value was deduced from the format of the image $(60 \times 60 \mu \mathrm{m})$

Figure S6: Diatoms grown with and without Phosphate: Increase in lipid droplets size and numbers in medium depleted in Phosphate

\section{AUTHOR CONTRIBUTIONS.}

M.B. performed, and analyzed the biological experiments and wrote the manuscript. C.B. built, characterized the optical setup and analyzed the movies and wrote the manuscript. HB and FC compared interferometry and fluorescence. SL and RD built the GFP fusions. FC and SD performed the culture of $P$ tricornutum and studied their growth under stress. RD, HCC and $\mathrm{CB}$ contibute the writing of the manuscript. All authors discussed the results and commented on the manuscript. 


\section{REFERENCES}

Abida, H., Dolch, L.J., Mei, C., Villanova, V., Conte, M., Block, M.A., Finazzi, G., Bastien, O., Tirichine, L., Bowler, C., Rebeille, F., Petroutsos, D., Jouhet, J. \& Marechal, E. (2015). Membrane glycerolipid remodeling triggered by nitrogen and phosphorus starvation in Phaeodactylum tricornutum. Plant Physiology, 167:118-136.

Alboresi, A., Perin, G., Vitulo, N., Diretto, G., Block, M., Jouhet, J., Meneghesso, A., Valle, G., Giuliano, G., Marechal, E. \& Morosinotto, T. (2016). Light remodels lipid biosynthesis in Nannochloropsis gaditana by modulating carbon partitioning between organelles. Plant Physiology, 171: 2468-2482.

Alipanah, L., Rohloff, J., Winge,P., Bones, A.M. \& Brembu,T. (2015) Whole-cell response to nitrogen deprivation in the diatom Phaeodactylum tricornutum. Journal of experimental botany,. 66: 6281-6296.

Alipanah, L., Winge, P., Rohloff, J., Najafi, J., Brembu, T. \& Bones, A.M. (2018) Molecular adaptations to phosphorus deprivation and comparison with nitrogen deprivation responses in the diatom Phaeodactylum tricornutum. PLoS ONE, 13: e0193335.

Apelian, C., Harms, F., Thouvenin, O. \& Boccara, C. (2016) Dynamic full field optical coherence tomography: subcellular metabolic contrast revealed in tissues by interferometric signals temporal analysis. Biomedical Optics Express, 7: 1511.

Apt, K.E., Zaslavkaia, L., Lippmeier, J.C., Lang, M., Kilian, O., Wetherbee, R., Grossman, A.R. \& Kroth, P.G. (2002). In vivo characterization of diatom multipartite plastid targeting signals. Journal of Cell Science, 115: 4061-406

Bailleul, B., Cardol, P., Breyton, C. \& Finazzi, G. (2011) Electrochromism: a useful probe to study algal photosynthesis. Photosynthesis Research, 106:179-89. Erratum in: Photosynthesis Research, 10:151-2.

Bowler, C., Vardi, A. \&Allen, A.E. (2010). Oceanographic and biogeochemical insights from diatom genomes. Annual Review of Marine Science, 2: 333-365.

Cavalier-Smith, T. (1999). Principles of Protein and Lipid Targeting in Secondary

Symbiogenesis: Euglenoid, Dinoflagellate, and Sporozoan Plastid Origins and the Eukaryote Family Tree. Journal of Eukaryotic Microbiology 46: 347-366.

Cruz de Carvalho, H., Sun, H.X., Bowler, C. \& Chua N.H. (2016). Noncoding and coding transcriptome responses of a marine diatom to phosphate fluctuations. New Phytologist, 210: $497-510$

De Martino, A., Meichenin, A., Shi, J., Pan, K. \& Bowler C. (2007) Genetic and phenotypic characterization of Phaeodactylum tricornutum (Bacillariophyceae) accessions. Journal of Phycology, 43: 992-1009.

Domingues, N., Matos, A.R., Marques da Silva, J. \& Cartaxana, P. (2012) Response of the Diatom Phaeodactylum tricornutum to Photooxidative Stress Resulting from High Light Exposure. PLoS ONE, 7: e38162. 
Falciatore, A, Casotti, R., Leblanc, C., Abrescia, C. \& Bowler, C. (1999). Transformation of Nonselectable Reporter Genes in Marine Diatoms. Marine Biotechnoly (NY), 1:239-251.

Falciatore, A., Jaubert, M., Bouly, J-P, Bailleul, B. \& Mock, T. (2020). Diatom Molecular Research Comes of Age: Model Species for Studying Phytoplankton Biology and Diversity. The Plant Cell, 32: 547-572.

Flori, S., Jouneau, P.H., Finazzi, G., Maréchal,E., \& Falconet D. (2016) Ultrastructure of the Periplastidial Compartment of the Diatom Phaeodactylum tricornutum. Protist, 167:254-267.

Gao, X., Bowler, C. \& Kazamia, E. (2021) Iron metabolism strategies in diatoms. Journal of Experimental Botany, 72: 2165-2180.

Goss, R., Wilhelm, C. \& Jakob, T. (2020). Photosynthesis in diatoms in Handbook of Algal Science, Technology and Medicine, 13:217-229.

Gould, S.B., Sommer M.S., Kroth P.G., Gile G.H., Keeling P.J. \& Maier U.G. (2006). Nucleus-to-Nucleus Gene Transfer and Protein Retargeting into a Remnant Cytoplasm of Cryptophytes and Diatoms. Molecular Biology and Evolution, 23:2413-2422.

Jacquier, N., Choudhary, V., Mari, M., Toulmay, A., Reggiori, F. \& Schneiter, R. (2011). Lipid droplets are functionally connected to the endoplasmic reticulum in Saccharomyces cerevisiae. Journal of Cell Science, 124: 2424-2437.

Jaussaud, A., Lupette, J., Salvaing, J., Jouhet, J., Bastien, O., Gromova, M. \& Maréchal E. (2020) Stepwise Biogenesis of Subpopulations of Lipid Droplets in Nitrogen Starved Phaeodactylum tricornutum Cells. Frontiers in Plant Science, 11:48.

Kazamia, E., Sutak, R., Paz-Yepes, J., Dorrell, R.G., Rocha Jimenez Vieira, F., Mach, J., Morrissey, J., Leon, S., Lam, F., Pelletier, E., Camadro, J-M., Bowler, C. \& Lesuisse, E. (2018) Endocytosis-mediated siderophore uptake as a strategy for Fe acquisition in diatoms Science Advances, 4: eaar4536.

Kolber , Z., Barber, R., Coale, K. et al. (1994) Iron limitation of phytoplankton photosynthesis in the equatorial Pacific Ocean. Nature, 371:145-149.

Leyland, B., Boussiba, S. \& Khozin-Goldberg, I. (2020) A Review of Diatom Lipid Droplets. Biology (Basel) 9:38.

Liang, Y., Koester, J.A., Liefer, J.D, Irwin, A.J. \& Finkel, Z.V. (2019) Molecular mechanisms of temperature acclimation and adaptation in marine diatoms. The ISME Journal, 13:2415-2425.

Lupette, J., Jaussaud, A., Seddiki, K., Morabito, C., Brugière, S., et al. (2019). The architecture of lipid droplets in the diatom Phaeodactylum tricornutum. Algal Research Biomass, Biofuels and Bioproducts, (Elsevier ed.) 38:101415.

Nettebrock, N.T. \& Bohnert, M. (2020) Born this way - Biogenesis of lipid droplets from specialized ER subdomains. BBA - Molecular and Cell Biology of Lipids, 1865:158448. 
Olzmann, J.A. \& Carvalho, P. (2019). Dynamics and functions of lipid droplets. Nature Review of Molecular Cell Biology, 20:137-155.

Pierella Karlusich, J. J., Ibarbalz, F. M.,\& Bowler, C. (2020). Phytoplankton in the Tara Ocean. Annual Review of Marine Science, 12: 233-265.

Prins, A., Deleris, P., Hubas, C. \& Jesus, B. (2020) Effect of Light Intensity and Light Quality on Diatom Behavioral and Physiological Photoprotection. Frontiers in Marine Science, 7:203

Roncel, M., González-Rodríguez, A.A., Naranjo, B., Bernal-Bayard, P., Lindahl, A.M., Hervás, M., Navarro, J.A. \& Ortega, J.M. (2016) Iron Deficiency Induces a Partial Inhibition of the Photosynthetic Electron Transport and a High Sensitivity to Light in the Diatom Phaeodactylum tricornutum. Frontiers in Plant Science, 7:1050.

Saunter, C.D., Perng, M., Love, G. \& Quinlan, R. (2009). Stochastically determined directed movement explains the dominant small-scale mitochondrial movements within non-neuronal tissue culture cells. FEBS Letters 583:1267-1273.

Scholler, J., Mazlin, V., Thouvenin, O., Groux, K., Xiao, P., Sahel, J.A., Fink, M., Boccara, C. \& Grieve, K. (2019). Probing dynamic processes in the eye at multiple spatial and temporal scales with multimodal full field OCT. Biomedical Optics Express, 10: 731-746.

Scholler, J. (2019) Motion artifact removal and signal enhancement to achieve in vivo dynamic full field OCT. Optics Express, 27: 19562-19572.

Schindelin, J., Arganda-Carreras, I., Frise, E., et al. (2012) Fiji: an open-source platform for biological-image analysis. Nature methods, 9: 676-682.

Thouvenin, O., Fink, M., \& Boccara C. (2017) Dynamic multimodal full-field optical coherence tomography and fluorescence structured illumination microscopy. Journal of Biomedical Optics, 22(2): 026004.

Thouvenin, O., Boccara, C., Fink, M., Sahel, J.A., Paques, M. \& Grieve, K. (2017) Cell motility as contrast agent in retinal explant imaging with full-field optical coherence tomography. Investigative Ophtalmology \& Visual Science, 58:4605.

Thouvenin,O., Alhaddad, S., Mazlin, V., Boccara, M., \& Boccara, C. (2021) Label free optical transmission tomography for biosystems: Intracellular structures and dynamics. arXiv 3991089.

Tominaga, M. \& iIto, K. (2015) The molecular mechanism and physiological role of cytoplasmic streaming. Current Opinion in Plant Biology, 27:104-110.

Ustick, L.J., Larkin, A.A., Garcia, C.A., Garcia, N.S., Brock, M.L., Lee, J.A., Wiseman, N.A., Moore, J.K. \& Martiny, A.C. (2021) Metagenomic analysis reveals global-scale patterns of ocean nutrient limitation. Science, 372: 287-291 
bioRxiv preprint doi: https://doi.org/10.1101/2021.10.22.465453; this version posted October 23, 2021. The copyright holder for this

preprint (which was not certified by peer review) is the author/funder, who has granted bioRxiv a license to display the preprint in perpetuity. It is made available under aCC-BY-NC-ND 4.0 International license.

Yao, S., Brandt, A., Egsgaard, H. and Gjermansen C. (2012) Neutral lipid accumulation at elevated temperature in conditional mutants of two microalgae species. Plant Physiology and Biochemestry, 61:71-79. 
Table 1: Effect of iron limitation after one (T1) and two (T2) passages on dynamic movements within Phaeodactylum tricornutum (Pt)

\begin{tabular}{|c|c|c|c|}
\hline & $\begin{array}{l}\text { number of cells } \\
10^{6} * \mathrm{~mL}^{-1} \pm \mathrm{SD}\end{array}$ & $\begin{array}{l}\text { Average number of } \\
\text { pixels with dynamic } \\
\text { behaviour } \pm \text { SD }\end{array}$ & $\begin{array}{l}\text { Number of } \\
\text { analysed cells }\end{array}$ \\
\hline + Fe T1 & $106 \pm 0.8$ & $1312 \pm 280$ & 39 \\
\hline$+\mathrm{Fe} \mathrm{T2}$ & $127 \pm 2$ & $1307 \pm 343$ & 36 \\
\hline - Fe T1 & $89 \pm 4$ & $1166 \pm 311^{\mathrm{a}}$ & 40 \\
\hline - Fe T2 & $10 \pm 0.8$ & $279 \pm 131^{b}$ & 46 \\
\hline
\end{tabular}


Table 2: Diameter and dynamic behaviour of droplets in full or phosphate-depleted medium.

\begin{tabular}{lll} 
& $\begin{array}{l}\text { Average droplet } \\
\text { diameter }(\mu \mathrm{m} \pm \mathrm{SD}) \\
\text { (number of analysed } \\
\text { droplets) }\end{array}$ & $\begin{array}{l}\text { Number of droplets } \\
\text { with dynamic } \\
\text { behaviour } \\
\text { (percentage) }\end{array}$ \\
+ Pi: exp1 & $0.56 \pm 0.22(42)$ & $22(52 \%)$ \\
- Pi: exp1 & $0.97 \pm 0.33(58)$ & $55(94 \%)$ \\
+ Pi: $\operatorname{exp2}$ & $0.43 \pm 0.11(18)$ & $6(33 \%)$ \\
- Pi: $\operatorname{exp2}$ & $0.96 \pm 0.32(26)$ & $21(80 \%)$ \\
+ Pi: $\operatorname{exp3}$ & $0.51 \pm 0.21(26)$ & $12(46 \%)$ \\
\hline Pi: $\operatorname{lexp3}$ & $0.88 \pm 0.22(86)$ & $61(71 \%)$
\end{tabular}


Table 3: Comparison of movements of the Phaeodactylum scatterers computing their standard deviation or cumulative sum in media with or without phosphate

\begin{tabular}{|c|c|c|c|}
\hline & $\begin{array}{l}\text { Number of } \\
\text { dynamic pixels } \\
\pm \text { SD (method: } \\
\text { standard } \\
\text { deviation) }\end{array}$ & $\begin{array}{l}\text { Number of } \\
\text { dynamic pixels } \\
\pm \text { SD (method: } \\
\text { Cumulative } \\
\text { sum) }\end{array}$ & $\begin{array}{c}\text { Ratio } \\
\text { Cumulative sum/ } \\
\text { Standard deviation }\end{array}$ \\
\hline$+\mathbf{P i}$ & $586 \pm 167$ & $1174 \pm 196$ & 2 \\
\hline$-\mathbf{P i}$ & $2844 \pm 155$ & $3311 \pm 236$ & 1.16 \\
\hline
\end{tabular}

a Three stacks have been analysed in each conditions 


\section{FIGURE LEGENDS}

\section{Figure 1: Effect of continuous or pulsed lighting on intracellular movements in Phaeodactylum tricornutum}

The cells were successively illuminated with the $\mathrm{LED}_{455}$, with either continuous or pulsed light and a film of few seconds recorded. The same field in the successive acquisition was selected; left image: transmission and right image: standard deviation (scale on right). A: pulsed lighting; B: continuous lighting. Histograms of the standard deviation images using image $\mathrm{J}$ (Fiji) were recorded and the number of pixels of each image were computed. A: histogram of standard deviation with pulsed lighting (cells: 5638 pixels with dynamic behaviour) and histogram with pulsed lighting (noise). B: histogram of standard deviation with continuous lighting (cells: 13 pixels with dynamic behaviour) and histogram with continuous lighting (noise). Scale bar value was deduced from the format of the image $(60 \times 60 \mu \mathrm{m})$.

Figure 2: Histogramm of pixels with dynamic behaviour: effects of wavelength illumination and photosystem II inhibitors.

The average values plus and minus standard deviation are presented. The different wavelengths tested are: $455 \mathrm{~nm}$ (blue), $505 \mathrm{~nm}$ (green) and 735nm (deep red). Two photosystem II inhibitors were used: DCMU (3,4-dichlorophenyl)-1,1-dimethyl-urea; $40 \mu \mathrm{M})$ and HA (hydroxylamine;2mM). 20 to 30 cells were analysed. A Wilcoxon test was realized the signal observed between $455 \mathrm{~nm}$ (blue) and $735 \mathrm{~nm}$ (deep red) and between $505 \mathrm{~nm}$ (green) and $735 \mathrm{~nm}$ (deep red) gave a pvalue of 0.0079 at a significance level of 0.05 )

The same test was realized with the photosystem II inhibitors: The signal observed between cntrol and DCMU or HA gave a pvalue of 0.03 at a significance level of 0.05

\section{Figure 3: Co-localisation of dynamic droplets from Phaeodactylum tricornutum and BODIPY@ labelled lipid droplets}

Left panel: transmission microscopy, central panel: fluorescence (grey scale camera, green coloration), right panel merge of the two previous images. Left panel: transmission microscopy, central panel: Standard deviation (grey scale camera, 16 colors coloration), right panel merge of the two previous images. Scale bar value was deduced from the format of the image $(90 \times 90 \mu \mathrm{m})$. 
Figure 4: Increase in lipid droplets size and numbers in $P$. tricornutum cells grown for 8 days in phosphate-depleted medium

From left to right panel (or from top to bottom) are shown, the transmission, the standard deviation of each pixel and the merged image (artificial blue colour). Histograms of the standard deviation images using image $\mathrm{J}$ (Fiji) were recorded and the number of pixels above noise level were computed (indicated by a red bar on the histogram). Histograms of the same image size without cells, were recorded to determine noise levels. Scale bar value was deduced from the format of the image $(60 \times 60 \mu \mathrm{m}$. 


\section{Pulsed light}
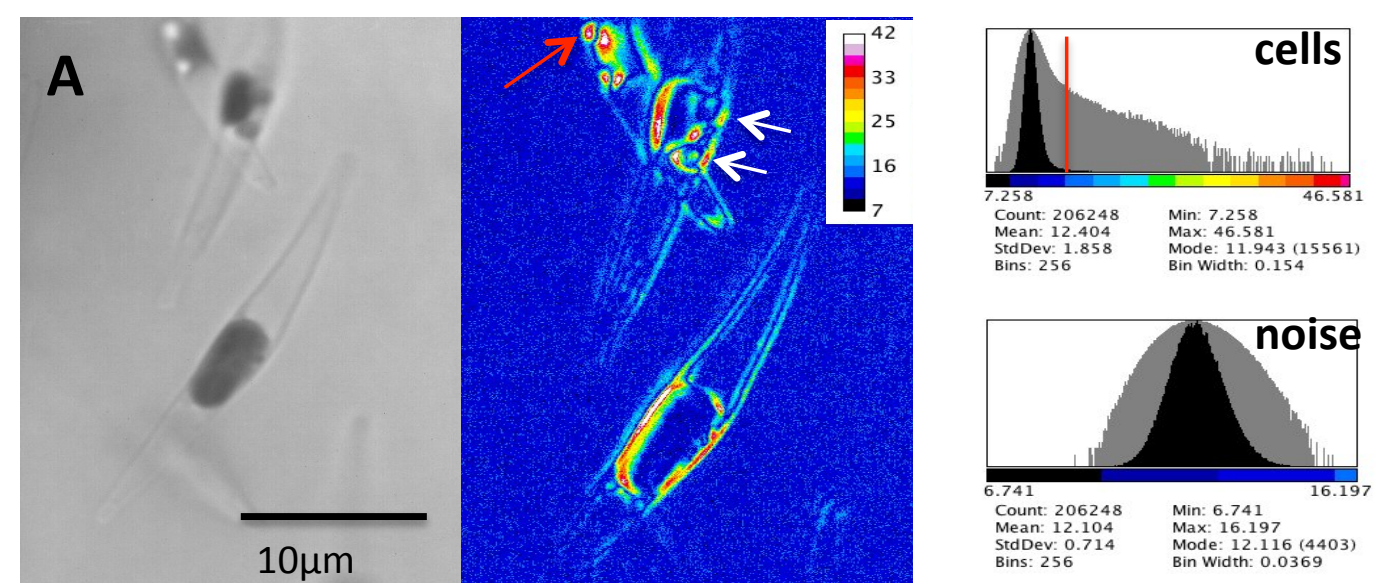

Figure 1

\section{Continuous light}
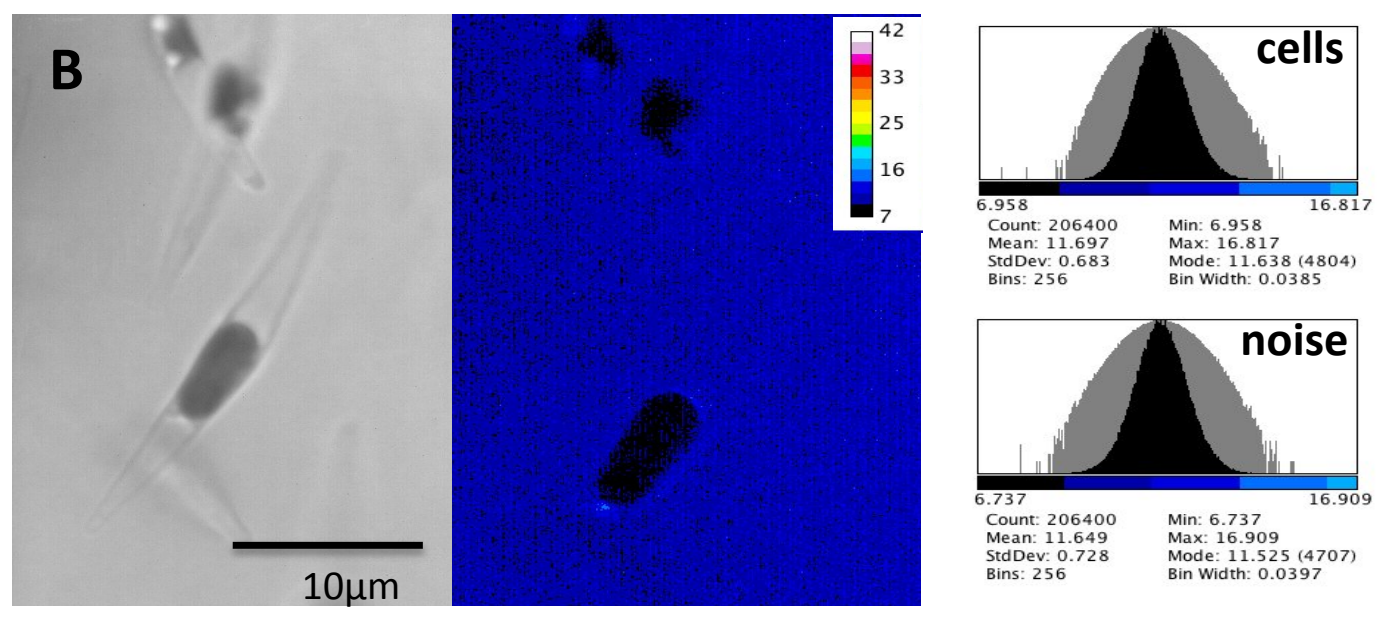
Figure 2

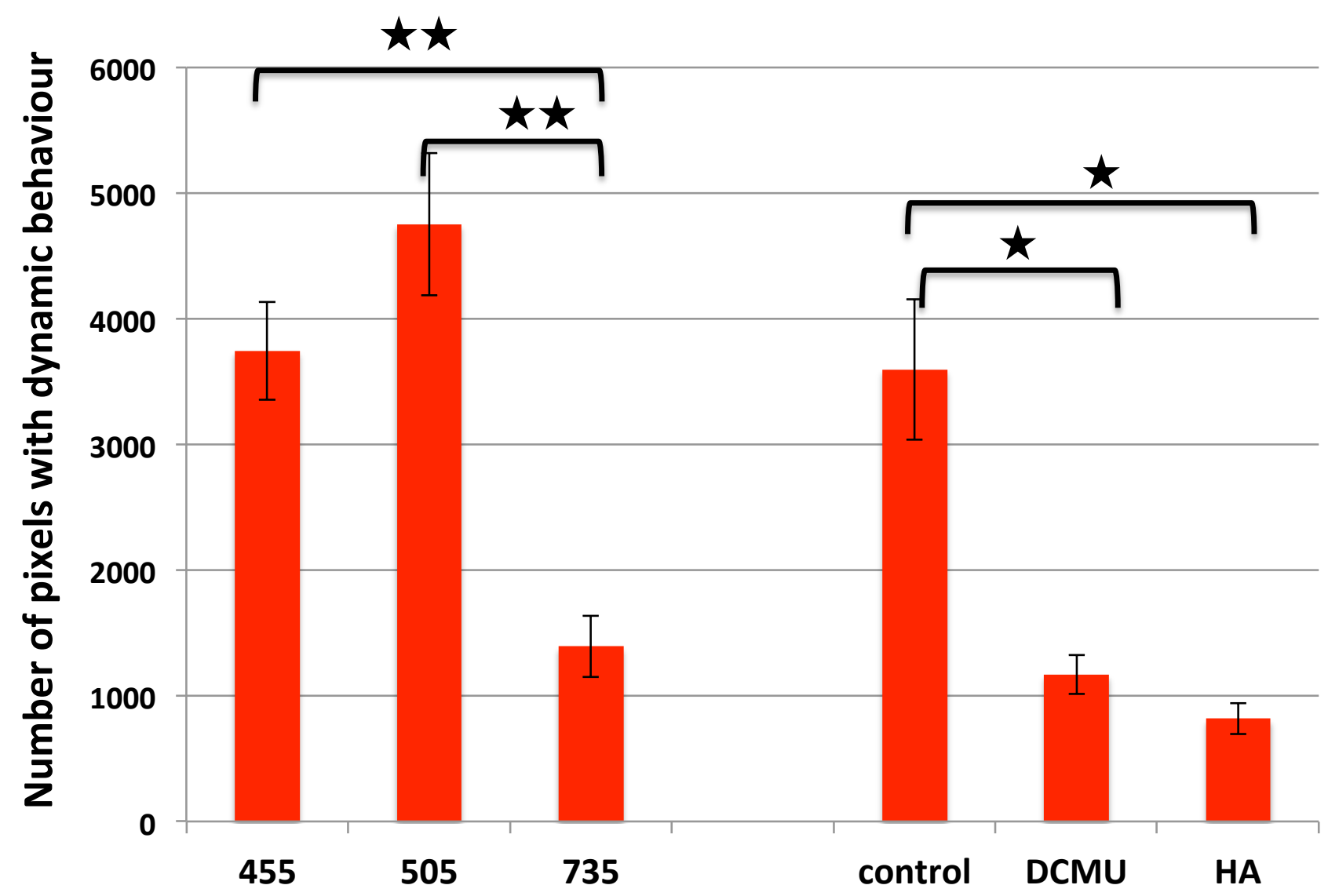



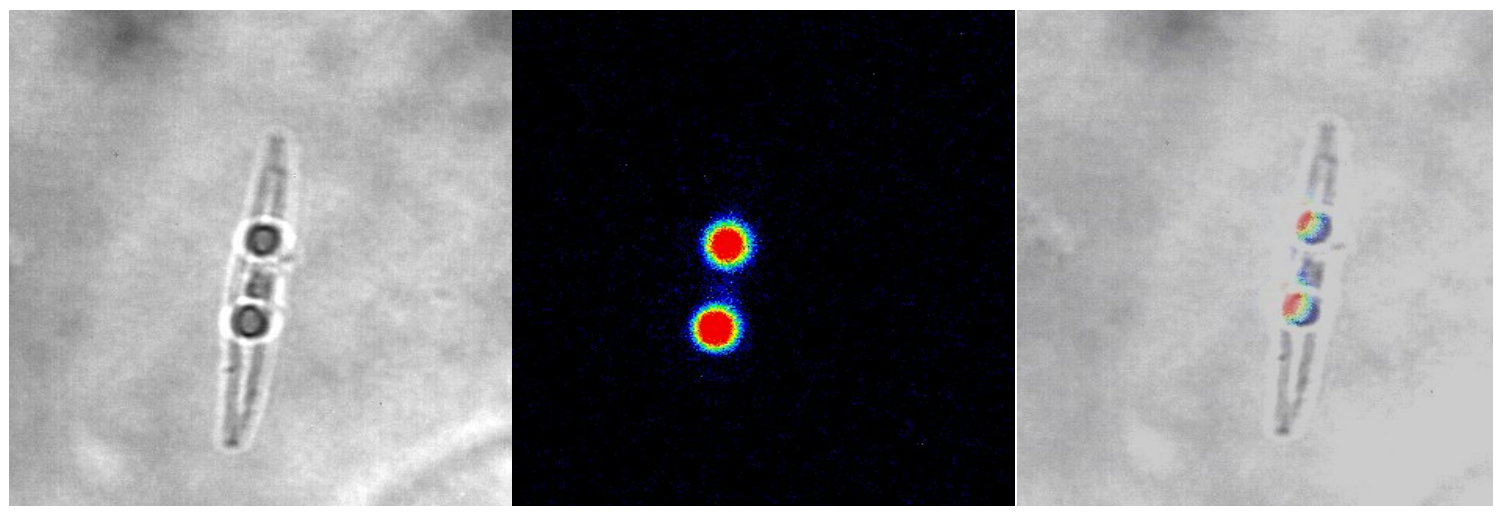

TRANSMISSION

DCI

MERGED
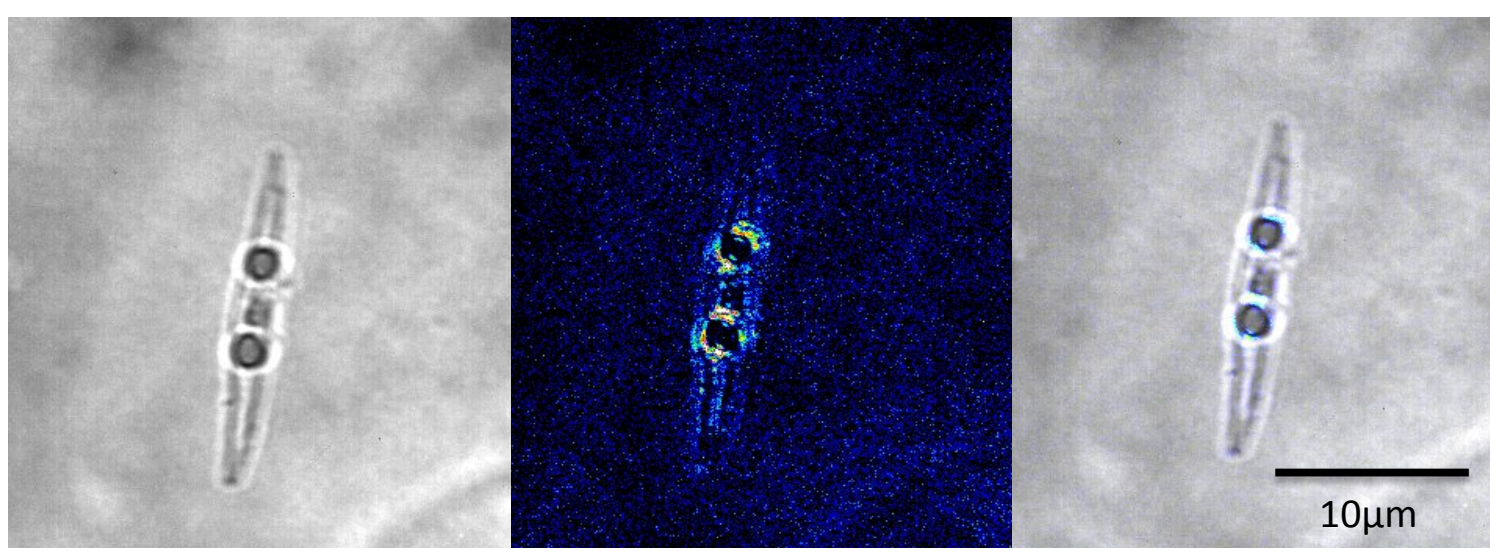
Figure 4

A

TRANSMISSION
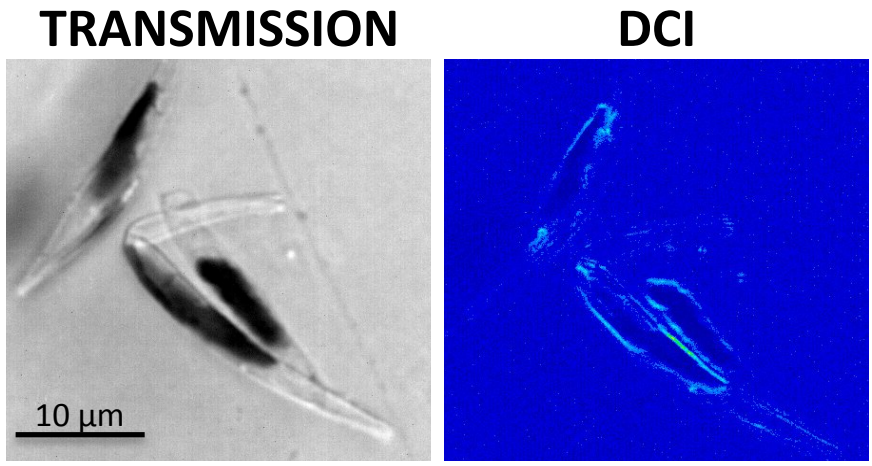

MERGED

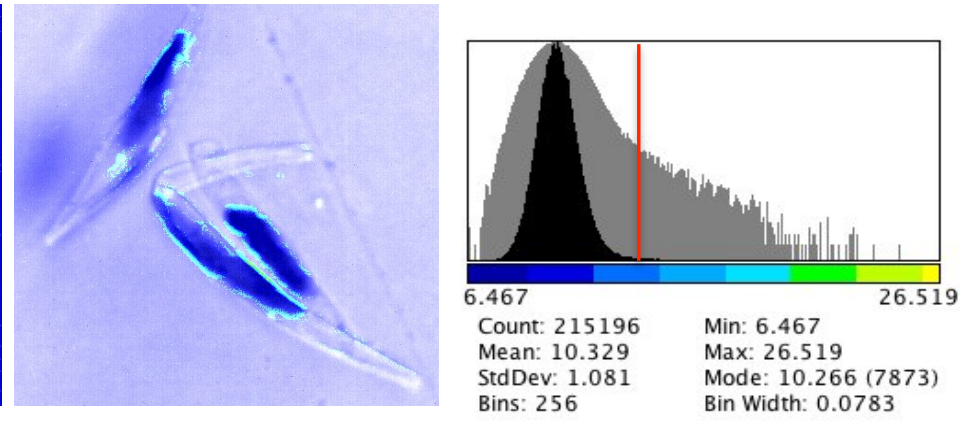

$+\mathrm{Pi}$

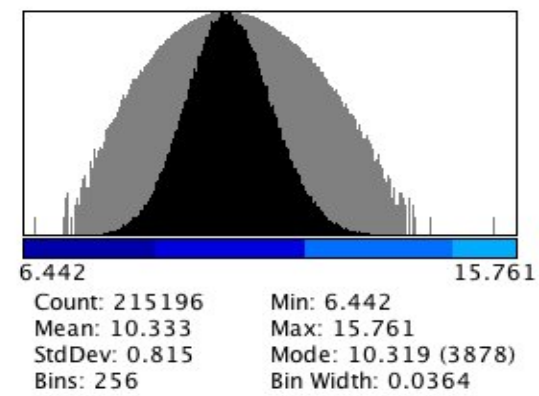

- Pi

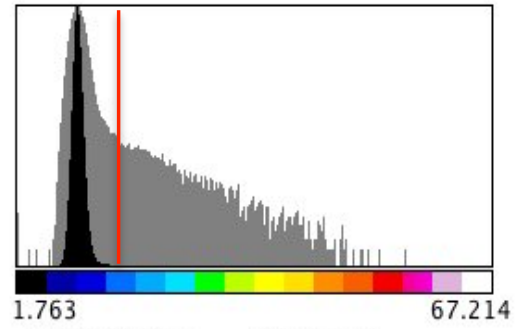

Count: 215136

Mean: 10.468

StdDev: 2.195

Max: 67.214

Mode: 10.072 (25841)

Bin Width: 0.256

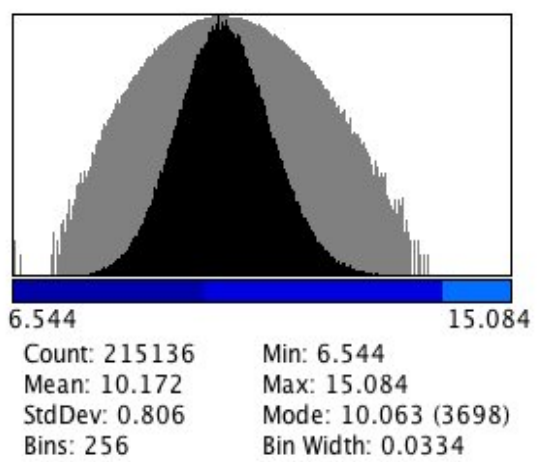

StdDev: 0.806 Bin Width: 0.0334

MERGED 